This is a postprint version of the following published document:

Pozuelo, J., López-González, M., Tlenkopatchev, M., Saiz, E. \& Riande, E. (2008): Simulations of gas transport in membranes based on polynorbornenes functionalized with substituted imide side groups. Journal of Membrane Science, 310 (1-2), pp.: 474-483.

DOI: 10.1016/j.memsci.2007.11.019

(C) Elsevier, 2008

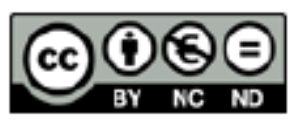

This work is licensed under a Creative Commons Attribution-NonCommercialNoDerivatives 4.0 International License. 


\title{
Simulations of gas transport in membranes based on polynorbornenes functionalized with substituted imide side groups
}

\author{
Javier Pozuelo ${ }^{\mathrm{a}}$, Mar López-González ${ }^{\mathrm{b}}$, Mikhail Tlenkopatchev ${ }^{\mathrm{c}}$, \\ Enrique Saiz ${ }^{\mathrm{d}}$, Evaristo Riande ${ }^{\mathrm{b}, *}$ \\ a Departamento de Ciencia e Ingeniería de Materiales e Ingeniería Química, Universidad Carlos III, Leganés, Madrid, Spain \\ ${ }^{\mathrm{b}}$ Instituto de Ciencia y Tecnología de Polímeros (CSIC), 28006 Madrid, Spain \\ ${ }^{\mathrm{c}}$ Instituto de Investigación en Materiales, Universidad Autónoma de México, Apartado Postal 70-360, Coyoacán, México, D.F. 04510, Mexico \\ ${ }^{\mathrm{d}}$ Departamento de Química-Física, Universidad de Alcalá, Alcalá de Henares, Madrid, Spain \\ * Corresponding author. Tel.: +34 91 5622900; fax: +34 91 5644853. E-mail address: riande@ @ictp.csic.es (E. Riande).
}

\begin{abstract}
This paper studies the diffusive and sorption steps of several gases across membranes cast from poly $(\mathrm{N}$-phenyl-exo,endonorbornene-5,6-dicarboximide) chloroform solutions. Chains packing effects on gas transport was investigated by conducting a parallel study on the permeation characteristics of membranes cast from hydrogenated poly( $N$-phenyl-exo,endo-norbornene-5,6dicarboximide) chloroform solutions. The perme-ability coefficients of several gases in the two membranes were measured finding that hydrogenation of the norbornene moieties decreases gas permeability. The transition states approach was used to determine the trajectories of the gases in the two types of membranes from which the diffusion coefficients were obtained. Monte Carlo techniques based on the Widom method were used to simulate gas sorption process as a function of pressure. The values of the solubility coefficients thus obtained undergo a relatively sharp drop at low pressures approaching to a constant value as pressure increases. With the exception of carbon dioxide, pretty good agreement between the experimental and simulated values of the permeability coefficient is found for the gases studied.
\end{abstract}

Keywords: Gas permeation; Diffusion simulation; Sorption simulation; Transition states approach; Widom method; Functionalized polynorbornenes

\section{Introduction}

In the last two decades, the search for better polymers as source of good performance membranes for gases separation has been a flourishing field of research [1,2]. As a first step, efforts have been focused on finding consistent relations between the structure of repeat units of the molecular chains integrating membranes and permeability coefficients, separation factors, etc. The screening of a large number of polymers having different chemical structures improves the chance of finding specific polymers with rather good transport properties without a refined understanding of the intrinsic properties leading to an adequate permselectivity. The success of this approach increases when a series of polymers within a same class are studied, noting the effect of small changes in structure on gas transport. It has been found that restriction of both packing efficiency and chain mobility may increase gas permeability with minimum permselectivity losses. Families of polycarbonates [3-9], polysulfones [4,10-17], polyimides [18-25], polyoxydiazoles and polytriazoles [26,27], aromatic polyesters [28-32], and methyland phenyl-substituted polyphenylene ethers [33,34], have been optimized using these guidelines. Work has been reported showing that the substitution of hydrogen atoms by methyl groups in the four symmetrical ring positions of bisphenol A leads to polycarbonates, polysulfones and polyarylates that are about four times more permeable to gases than the corresponding unsubstituted materials without losing permselectivity [33].

Another approach to the study of the influence of chemical structure on gas permeability is to simulate the trajectories of diffusing species in membranes by molecular dynamics $[35,36]$. Once steady-state regime is reached, the diffusion coefficient is obtained from the mean-square displacements of the diffu- 
sant. Full molecular dynamics is useful for membranes very permeable to gases, e.g. polydimethyl siloxane [37], or atactic polypropylene [38], where diffusive motion can be sampled in relatively short molecular dynamics simulations. However, this technique encounters serious difficulties while trying to simulate gas transport in glassy membranes, commonly utilized for gases separation, where only local molecular motions are permitted. Actually, a diffusant particle spends prohibitively long times wandering in the cavities that account for the excess volume in glassy systems until occasional fluctuations produce channels with suitable diameters through which the diffusant may slide to a nearby cavity. In this case the trajectory of the diffusant can be simulated using the transition states approach (TSA) described by Suter and co-workers [39]. In addition, methods based on the Widom method [40] have been developed [35,41,42] that allow the simulation of the sorption step in glassy membranes as a function of both pressure and chemical structure.

A wealth of experimental work has been done focused on gas transport in membranes based on polynorbornenes [43-53]. These polymers can be prepared via ring-opening metathesis polymerization (ROMP) of bicycloolefins, the latter being synthesized by $[4+2]$ cycloaddition of olefins to cyclopentadiene or to its derivatives. Tlenkopatchev et al. [54-57] took advantage of the facile functionalization of norbornene monomers and high reactivity in ring-opening metathesis polymerization to extend gas transport studies to membranes cast from polynorbornenes functionalized with substituted imide side groups. Thus new high $\mathrm{Tg}$ polymers have been obtained by ring-opening metathesis polymerization of exo,endo- $N-(1-\mathrm{R})$-norbornene5,6-dicarboximide using well-defined vinylidene ruthenium catalysts, where R can be phenyl, cyclohexyl, adamantyl groups, etc. The gas transport characteristics of membranes prepared from homopolymers or copolymers with different $\mathrm{R}$ groups anchored to the imide group of the comonomers have been reported [54-57].

The aim of this work is to study the effect of the increase of the flexibility of polynorbornene functionalized with imide side groups on the gas permeation characteristics of membranes cast from solutions of the corresponding polymers. To accomplish this goal, a parallel study comprising the experimental measurements and simulations of the diffusion and solubility coefficients of different gases (nitrogen, oxygen, carbon dioxide, carbon monoxide and methane) was carried out across a poly(exo,endo- $N$-phenyl-norbornene-5,6-dicarboximide) membrane (PNDCI) and its hydrogenated counterpart (H-PNDCI). Schemes of the respective polymers are shown in Fig. 1. Simulation of the diffusion coefficients of the gases was carried out using the TSA whilst that of the solubility coefficient was performed using an approach based on the Widom method [41,42]. The simulated parameters are compared with the experimental ones obtained from permeation curves.

\section{Experimental}

Poly( $N$-phenyl-exo,endo-norbornene-5,6-dicarboximide) (PNDCI) was obtained by ring-opening metathesis polymerization (ROMP) of exo-(60\%) and endo-(40\%) norbornene-
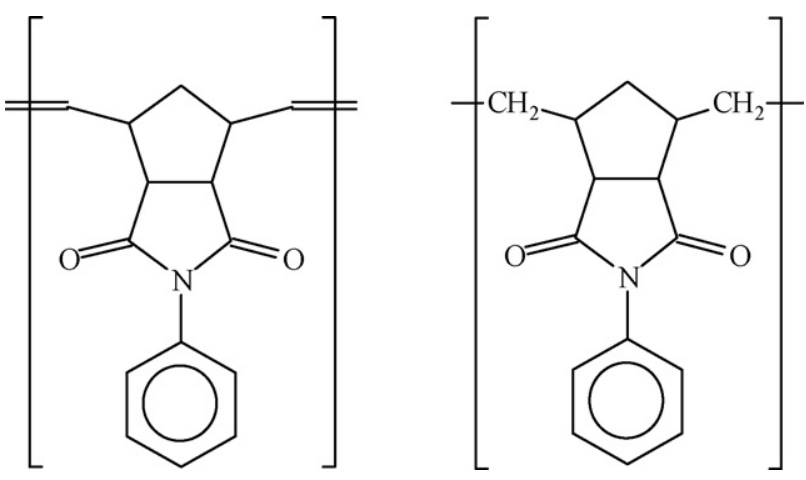

Fig. 1. Schematic representation of the repeating units of poly(exo,endo$\mathrm{N}$-phenyl-norbornene-5,6-dicarboximide) (PNDCI) and its hydrogenated counterpart (H-PNDCI).

5,6-dicarboxylic anhydride (NDA). The hydrogenation of poly( $N$-phenyl-exo,endo-norbornene-5,6-dicarboximide) was carried out at room temperature, under 115 bar, using the Wilkinson catalyst $\mathrm{CIRh}\left(\mathrm{PPh}_{3}\right)_{3}$. The hydrogenation yield was 99\%. Details of the synthesis and characterization of PNDCI and the hydrogenated polymer, H-PNDCI, are given elsewhere [57]. The values of the number average molecular weight $\left(M_{\mathrm{n}}\right)$, polydispersity $\left(M_{\mathrm{w}} / M_{\mathrm{n}}\right)$, glass transition temperature $\left(T_{\mathrm{g}}\right)$, and decomposition temperature $\left(T_{\mathrm{d}}\right)$, of H-PNDCI were, respectively, $2.3 \times 10^{5}, 1.6,197^{\circ} \mathrm{C}$ and $460{ }^{\circ} \mathrm{C}$.

\subsection{Gas permeation}

Gas transport in membranes cast from the respective PNDCI and H-PNDCI dichloroethane polymers solutions was measured in an experimental device made up of two compartments separated by the membrane. Vacuum was made in the two compartments and gas stored in a reservoir at the pressure of interest was allowed to flow into one of the compartments called hereafter upstream chamber. The flow of gas from the upstream chamber to the low pressure or downstream chamber was monitored with a MKS-722 (0-10 Torr) pressure sensor via a PC. The pressure in the upstream chamber was measured with a Gometrics $(0-10$ bar) pressure sensor. The gas permeation apparatus was immersed in a water thermostat.

\section{Results and discussion}

\subsection{Experimental results}

Illustrative curves describing the evolution of the pressure of gas in the downstream chamber, at $30^{\circ} \mathrm{C}$, for hydrogen, nitrogen, oxygen, carbon dioxide and methane are shown in Fig. 2. The isotherms present a transitory process followed by a linear dependence of pressure on time that reflects steady-state conditions. The time at which the extrapolated $p$ versus $t$ straight line intersects the abscissa axis, called time lag $\theta$, is further used for the determination of the gas diffusion coefficient. The permeability coefficient, $P$, was obtained from the slope of the isotherms in steady-state conditions by means of the following 


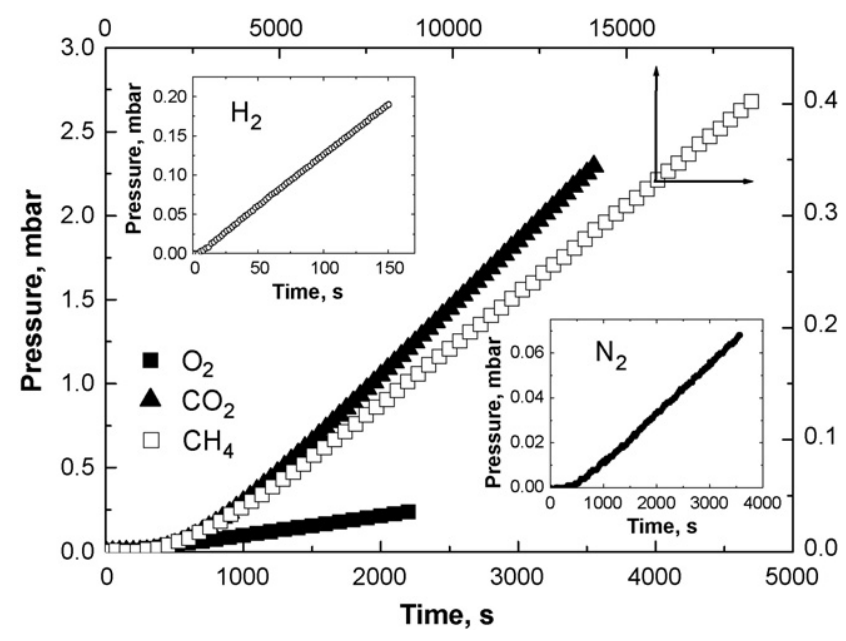

Fig. 2. Isotherms showing the evolution of pressure with time in the downstream chamber for hydrogen (inset), $\mathrm{N}_{2}$, carbon dioxide and methane.

expression:

$P=\frac{273}{76} \frac{V l}{p_{0} T A} \lim _{t \rightarrow \infty} \frac{\mathrm{d} p}{\mathrm{~d} t}$

where $p_{0}$ is the pressure in the upstream chamber, $V$ the volume of the downstream chamber, $T$ the absolute temperature, and $l$ and $A$ are, respectively, the thickness and permeation area of the membrane. The parameter $P$ is given in barrers $\left[1\right.$ barrer $\left.=10^{-10} \mathrm{~cm}^{3}(\mathrm{STP}) \mathrm{cm} /\left(\mathrm{cm}^{2} \mathrm{~s} \mathrm{~cm} \mathrm{Hg}\right)\right]$. The diffusion coefficient was obtained from [58]:

$D=\frac{l^{2}}{6 \theta}$

The apparent solubility coefficient, $S$, was calculated from

$S=\frac{P}{D}$

Usually, $D$ and $S$ are given, respectively, in $\mathrm{cm}^{2} / \mathrm{s}$ and $\mathrm{cm}^{3}(\mathrm{STP}) / \mathrm{cm}^{3} \mathrm{~cm} \mathrm{Hg}$.

Values of the permeation, diffusion and solubility coefficients for different gases in PNDCI and H-PNDCI membranes are shown in Tables 1 and 2, respectively. Owing to its very small molecular size, hydrogen presents the highest diffusion coefficient of all the gases. The values of $D$ for $\mathrm{H}_{2}$ in PNDCI and H-PNDCI are $1.32 \times 10^{-6}$ and $1.16 \times 10^{-6} \mathrm{~cm}^{2} / \mathrm{s}$, respectively. In spite of the fact that the diffusion coefficient of hydrogen in the membranes is nearly two orders of magnitude higher than

Table 1

Values of the experimental and simulated diffusion coefficient for PNDCI membranes at $30^{\circ} \mathrm{C}$

\begin{tabular}{lcc}
\hline Gas & $D_{\exp }\left(\times 10^{9} \mathrm{~cm}^{2} / \mathrm{s}\right)$ & $D_{\text {simul }}\left(\times 10^{9} \mathrm{~cm}^{2} / \mathrm{s}\right)$ \\
\hline $\mathrm{H}_{2}$ & 1320.0 & 2980.0 \\
$\mathrm{~N}_{2}$ & 22.3 & 10.8 \\
$\mathrm{O}_{2}$ & 63.0 & 16.1 \\
$\mathrm{CO}_{2}$ & 18.1 & 6.9 \\
$\mathrm{CO}$ & 14.5 & 10.4 \\
$\mathrm{CH}_{4}$ & 7.2 & 5.8
\end{tabular}

Table 2

Values of the experimental and simulated diffusion coefficient for H-PNDCI membranes at $30^{\circ} \mathrm{C}$

\begin{tabular}{lcc}
\hline Gas & $D_{\exp }\left(\times 10^{9} \mathrm{~cm}^{2} / \mathrm{s}\right)$ & $D_{\text {simul }}\left(\times 10^{9} \mathrm{~cm}^{2} / \mathrm{s}\right)$ \\
\hline $\mathrm{H}_{2}$ & 1164 & 1691 \\
$\mathrm{~N}_{2}$ & 9.4 & 10.1 \\
$\mathrm{O}_{2}$ & 31.1 & 15.2 \\
$\mathrm{CO}_{2}$ & 7.2 & 2.2 \\
$\mathrm{CO}$ & 7.6 & 5.3 \\
$\mathrm{CH}_{4}$ & 1.8 & 3.2
\end{tabular}

that of carbon dioxide, the permeability of both gases across the membranes is nearly similar. Actually the values of the permeability coefficient for $\mathrm{H}_{2}$ and $\mathrm{CO}_{2}$ in PNDCI are 11.0 and 11.4 barrers, respectively. In the H-PNDCI membrane, these values drop to 7.2 and 4.5 barrers, respectively. Obviously, the high solubility of $\mathrm{CO}_{2}$ in comparison with that of $\mathrm{H}_{2}$ compensates its lower diffusivity and, as a result, the values of the permeability coefficients of the two gases are rather close.

In general, the values of the diffusion coefficient of the gases are lower in the H-PNDCI membrane than in the PNDCI one presumably as a consequence of the fact that hydrogenation of the double bonds of PNDCI chains facilitates chain packing causing the diminution of the diffusion coefficient. On the other hand, the results suggest that an increase in chain packing efficiency has a minor effect on the solubility of the gases in the membranes.

\subsection{Simulation of gas diffusion in PNDCI and H-PNDCI glassy membranes}

From the physics of Brownian motion, a relationship between the diffusion coefficient of a diffusive particle, $D$, and its average mean-square displacement, $\mathbf{r}$, at time $t$ is easily obtained. The pertinent expression, called Einstein's equation, is given by

$D=\frac{1}{6} \lim _{t \rightarrow \infty}\left\{\frac{\delta}{\delta t}\left\langle(\mathbf{r}(t)-\mathbf{r}(0))^{2}\right\rangle\right\}$

where $\mathbf{r}(t)$ is the vector position of the particle at time $t$ in the laboratory reference frame and the symbol $\langle\cdots\rangle$ means average.

Trajectories of the gases in the membranes were simulated using the transition state approach [59-64] that involves three steps: (a) simulation of the polymer matrix by packing the chains in a virtual box; (b) evaluation of the probability density function that describes the local minima of energy, called sites, and the crest surfaces between adjacent sites in the matrix; (c) Monte Carlo simulation of random walks of the guest molecule within the polymer matrix.

In the first step, five chains, each one of them containing 15 repeat units of the corresponding polymer (PNDCI or HPNDCI), were generated and packed into a cubic box of side length about $30 \AA$ with periodic boundary conditions (PBC) to provide a density of approximately $1.20 \mathrm{~g} / \mathrm{cm}^{3}$ for PNDCI close to the value of $1.24 \mathrm{~g} / \mathrm{cm}^{3}$ found from pycnometric measurements. Minimization of conformational energy of the polymer in the box was accomplished using the Materials Studio 3.2 [65] molecular modelling package and the pcff91 [66]. The van der 
Waals and Coulombic non-bonding interactions were calculated by atom based and Ewald methods [67], respectively. Annealing cycles were simulated by MD trajectories that heated the lattice from 300 to $2000 \mathrm{~K}$ and then cooled it down back to $300 \mathrm{~K}$. Both the heating and cooling processes were carried out with $50 \mathrm{~K}$ increments and $1 \mathrm{ps}$ time step. In this way any dependence on the initial conformation was eliminated. The energy of the last conformation obtained by annealing was minimized with respect to bond lengths, bond angles and rotations using NVT and NPT dynamics of 500 and $100 \mathrm{ps}$, respectively, at $300 \mathrm{~K}$.

In the second step, each side of the cubic box was divided in $G=100$ intervals thus obtaining a grid containing $10^{6}$ cells. The partition function and free energy arising from placing a diffusant molecule in a randomly chosen cell was computed using a protocol which assumes that each atom of the polymer matrix is fluctuating about its mean position with a root mean-square deviation, $\Delta$, known as smearing factor [39]. The probability of finding an atom $i$ of the polymer matrix at a distance $\delta$ from the MD computed main position is given by

$W(\delta)=\exp \left(\frac{-\delta^{2}}{2 \Delta^{2}}\right)$

Obviously, the probability depends on the magnitude of the displacement $\delta$, but not on its direction.

Assuming that a molecule of diffusant gas is located in cell $m$ of the polymeric matrix, representing by $\mathbf{R}_{m}$ the vector joining the center of masses of the gas molecule with the main position of the matrix $i$ atom and by $\boldsymbol{\delta}_{i}$ the vector joining the main and instantaneous positions of atom $i$ (i.e. $\boldsymbol{\delta}_{i}$ has a module $\delta$ and a direction governed by two angles $\theta$ and $\phi$ ) the interaction between the matrix atom and the diffusant molecule will depend on $\mathbf{r}=\mathbf{R}_{m}+\boldsymbol{\delta}_{i}$. In previous publications we have treated polyatomic molecules as separated atoms, so that the energy was computed as sum of interactions of the matrix atom with each one of the atoms of the diffusant molecule and averaged over all orientations that the diffusant may adopt through rotations over its center of mass $[41,42,63,64,68]$. However, in the present paper we represent all the diffusant molecules by spherical particles with energetic parameters adjusted in such a way that they produce the same interaction that would be obtained by addition over all the atoms in the molecule and average over all the positions. These averaged parameters optimized for pcff force field are collected in Table 3. Kucukpinar et al. [69] used this parameters in permeability study of these gases in copolymers of styrene, Fried et al. in poly(2,6-dimethyl-1,4-phenylene oxide)

Table 3

Lennard-Jones $(6,9)$ parameters employed to compute the interaction between polymer matrix and diffusant gas molecules appearing in Eqs. (6) and (7)

\begin{tabular}{llr}
\hline Difussant & $\sigma(\AA)$ & $\varepsilon(\mathrm{K})$ \\
\hline $\mathrm{H}_{2}$ & 2.93 & 37.0 \\
$\mathrm{~N}_{2}$ & 3.70 & 95.1 \\
$\mathrm{O}_{2}$ & 3.46 & 118.0 \\
$\mathrm{CO}_{2}$ & 3.70 & 226.5 \\
$\mathrm{CO}$ & 3.70 & 111.4 \\
$\mathrm{CH}_{4}$ & 3.89 & 199.3
\end{tabular}

[70] and Heuchel et al. in polyimides [71]. Good agreement between simulated and experimental results was found.

Representing by $E\left(\mathbf{R}_{m}, \delta, \theta, \phi\right)$ [39] the interaction between the matrix $i$ atom having a displacement $\boldsymbol{\delta}_{i}$ (modulus $\delta$ and orientation defined by the angles $\theta$ and $\phi$ ) from its main position and the whole diffusant molecule placed at grid position $m$, the probability of finding such an arrangement of matrix atom and diffusant molecule would be

$W_{i}\left(R_{m}, \delta, \theta, \phi\right)=C \exp \left(\frac{-\delta^{2}}{2 \Delta^{2}}\right) \exp \left(\frac{-E_{i}\left(R_{m}, \delta, \theta, \phi\right)}{k T}\right)$

where $C$ represents a normalization constant. The partition function arising from interactions between the diffusant molecule in the cell $m$ and the atom $i$ of the matrix that is fluctuating around its main position, can be written as

$$
\begin{aligned}
Z_{m, i}\left(R_{m}\right)= & C \int_{0}^{\pi} \sin \theta \mathrm{d} \theta \int_{0}^{2 \pi} \mathrm{d} \phi \int_{0}^{\delta_{\max }} \delta^{2} \\
& \times \exp \left(\frac{-\delta^{2}}{2 \Delta^{2}}\right) \exp \left(\frac{-E_{i}\left(R_{i}, \delta, \theta, \phi\right)}{k T}\right) \mathrm{d} \delta
\end{aligned}
$$

Then the partition function of the cell $m$ containing a diffusant molecule which interacts with the whole polymer matrix is obtained through multiplication of one factor similar to Eq. (6) for each atom of the polymer matrix, i.e.

$Z_{m}=\prod_{i=1}^{n} Z_{m, i}$

where $n$ is the number of atoms of the polymer matrix in the cubic box.

The change of free energy associated with the insertion of a molecule of diffusant in a grid position $m$ is straightforwardly obtained from $Z_{m}$ as

$\Delta F_{m}=-k T \ln Z_{m}$

After computing $\Delta F_{m}$ for each one of the grid cells of the lattice, the so-called sites, or local minima of the free energy of the matrix, were located by a steepest descent algorithm starting at each grid point that ends into the site where the starting grid point belongs. The partition functions for the site $i, Z_{i}$, and the crest between sites $i$ and $j, Z_{i j}$, cover the grid points defining the valley and the crest, respectively. These functions can be written as

$Z_{i}=\left(\frac{L}{G}\right)_{k}^{3}\left(\frac{-\Delta F_{k}}{k T}\right)$

$Z_{i j}=\left(\frac{L}{G}\right)_{k}^{2} \gamma_{k}\left(\frac{-\Delta F_{k}}{k T}\right)$

where $L$ is the side length of the PBC box and $N$ is the number of intervals in which this side has been divided for the preparation of the grid. The weighting factor $\gamma_{m}$ in Eq. (11) arises from the fact that the crest surface between sites $i$ and $j$ is defined by the loci of grid points $k$ meeting the condition that $k$ belongs to 
site $i$ whereas at least one among its next neighbors belongs to site $j$. The weighting factor is defined as $\gamma_{k}=1,2^{1 / 2}, 1.41,0$, respectively, when one, two, three or four among the six next neighbors of grid point $m$ belongs to site $j$. The rate of passage from site $i$ to site $j, R_{i j}$, is given by

$R_{i j}=\left(\frac{k T}{8 \pi m}\right)^{1 / 2} \frac{Z_{i j}}{Z_{i}}$

whilst the residence time of the diffusant in the site $i, \tau_{i}$, is

$\tau_{i}=\frac{1}{\sum_{j=1}^{n} R_{i j}}$

The trajectory of the diffusant in the polymer matrix was computed by randomly choosing site $i$ and one of its neighbors, $j$, as initial and final sites of the first jump. The residence time of the diffusant molecule in site $i$ is obtained by means of Eq. (13). The simulation continues by taking position $j$ as initial position for a second jump to one of its neighbor sites and so on until the accumulated time equals a predetermined value. The square of the displacement of the diffusant from the initial position $(\Delta r)^{2}$ is recorded as a function of time.

A rough analysis involving thermal energy, bond stretching and bond angle bending force constants suggests that the smearing factor $\Delta$ in Eq. (5) should lie in the range $0.3-0.4 \AA$. The latter value was used in the simulation of the trajectories of the gases in the polymer matrix. The average of the MSD of 500 independently trajectories generated for each one of the following gases: oxygen, nitrogen, carbon dioxide, carbon monoxide and methane in PNDCI and H-PNDCI are plotted as a function of time in Figs. 3 and 4, respectively. The two figures show the variation of the MSDs with time for hydrogen which are much higher than those found for the other gases. The variation with time of the diffusion coefficient of the gases in PNDCI and H-PNDCI, obtained from the trajectories by means of Eq. (4), are shown in Figs. 5 and 6, respectively. The curves undergo a sharp drop at short times until the diffusive regime is reached at which the values of $D$ apparently remain constant. The drop of

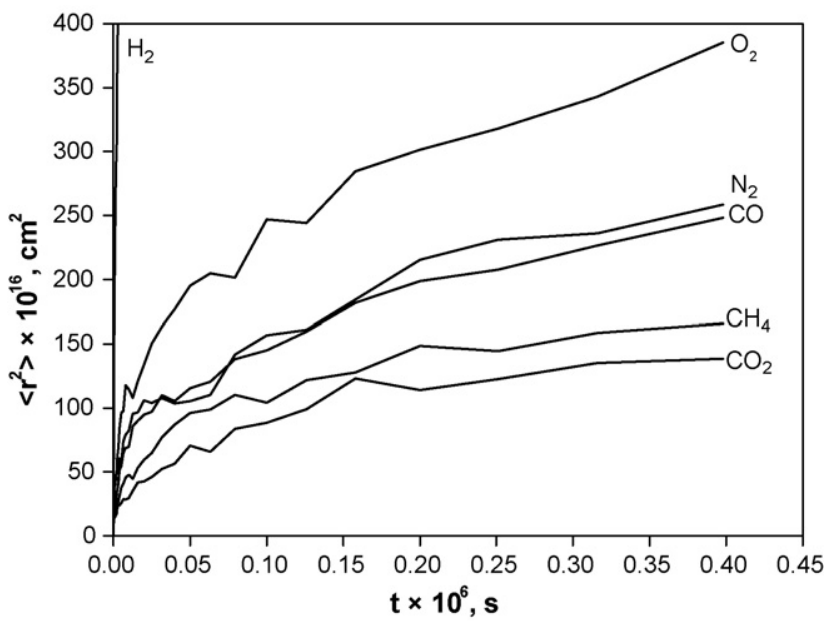

Fig. 3. Plots of mean-square displacements vs. time for different gases in PNDCI membranes, at $30^{\circ} \mathrm{C}$ and 1 bar.

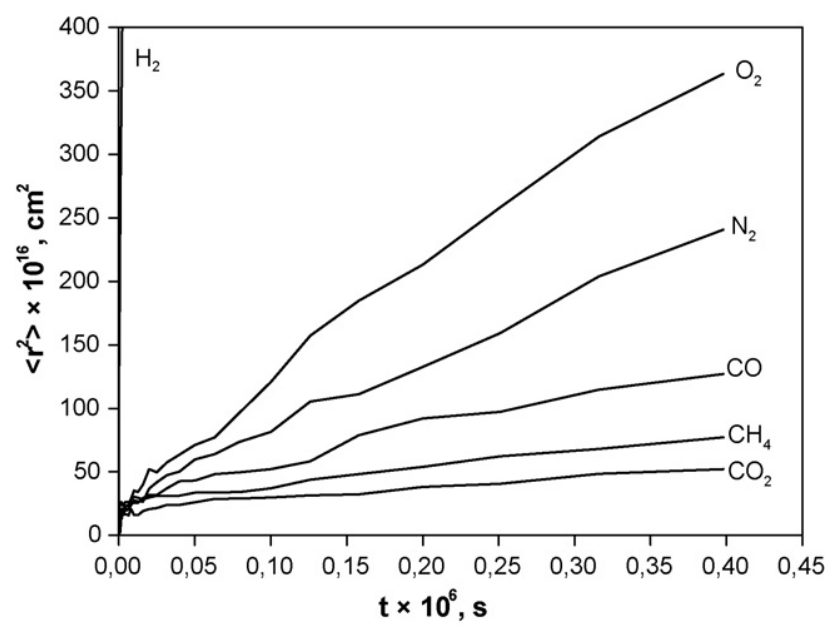

Fig. 4. Time dependence of the mean-square displacements for different gases in $\mathrm{H}$-PNDCI membranes, at $30^{\circ} \mathrm{C}$ and 1 bar.

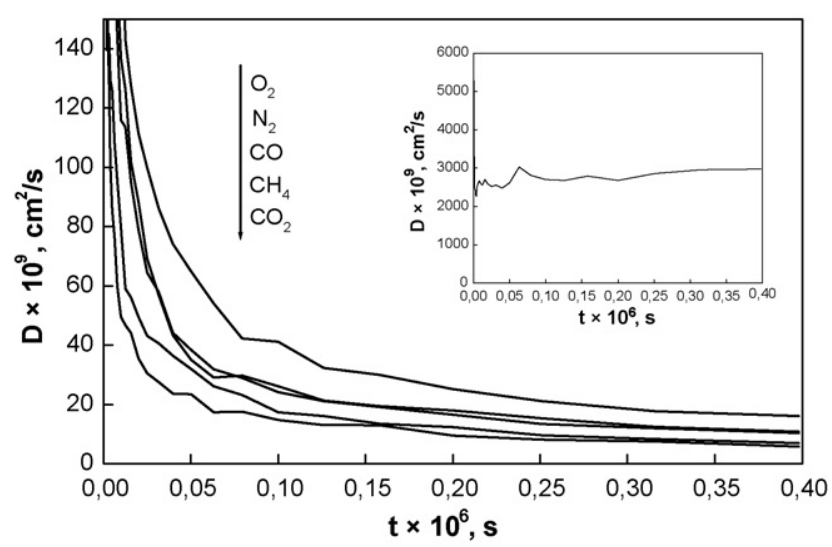

Fig. 5. Variation of the simulated diffusion coefficient with time for different gases in PNDCI, at $30^{\circ} \mathrm{C}$ and 1 bar. Inset: time dependence of the simulated diffusion coefficient for hydrogen.

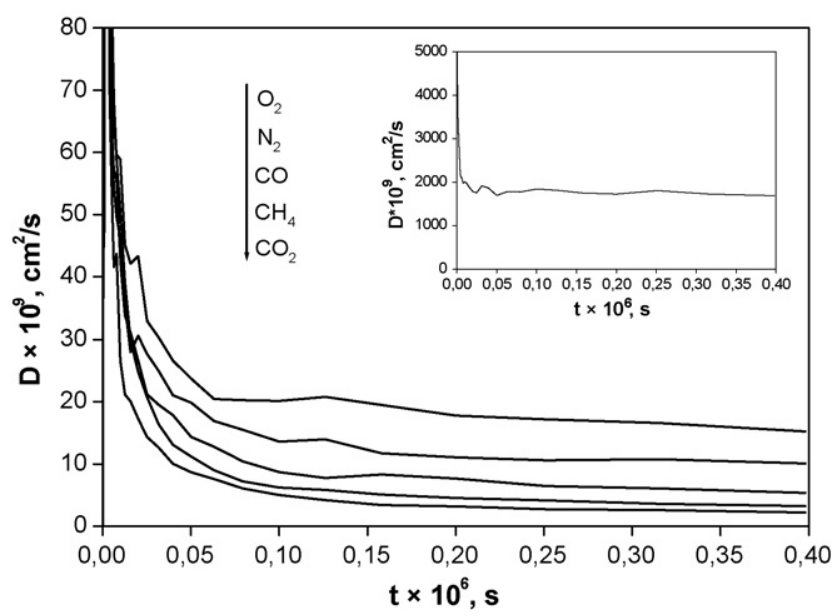

Fig. 6. Time dependence of the diffusion coefficient for different gases in $\mathrm{H}$ PNDCI membranes, at $30^{\circ} \mathrm{C}$ and 1 bar. Inset: time dependence of the simulated diffusion coefficient for hydrogen. 
$D$ at short times presumably is a consequence of poor statistical sampling.

The values simulated for the diffusion coefficient of the gases in PNDCI and H-PNDCI matrices are shown in Tables 1 and 2, respectively. With the exception of carbon dioxide, the simulated results for the diffusion coefficients of the gases studied in this work follow the same trends observed in the experimental ones in the sense that $D\left(\mathrm{H}_{2}\right) \gg D\left(\mathrm{O}_{2}\right)>D\left(\mathrm{~N}_{2}\right)>D(\mathrm{CO})>D\left(\mathrm{CH}_{4}\right)$. Moreover not only the experimental and simulated values are of the same order of magnitude, but also are rather close in most cases. For example, the simulated value for the diffusion coefficients of $\mathrm{H}_{2}$ in the PNDCI and H-PNDCI membranes are, respectively, $125 \%$ and $45 \%$ higher than the experimental ones. At the other extreme, the simulated values of $D$ for $\mathrm{CO}$ are about $30 \%$ lower than the experimental ones. Moreover the simulated values of the diffusion coefficients of most gases in PNDCI are higher than those simulated in H-PNDCI membranes. The influence of chains packing efficiency on hindering the diffusive step is better reflected in the simulated diffusion coefficient of carbon dioxide which drops from 6.94 to 2.18 in $10^{-9} \mathrm{~cm}^{2} / \mathrm{s}$ units.

Simulations of the solubility process were performed employing a modified Widom method consisting on series of insertion/removal of gas molecules into/from the polymer matrix according to probabilities computed by taking into account both energetic and geometrical factors $[41,42]$. We thus assume that the polymer matrix contains $n$ molecules of the diffusant gas and we try to insert a new one (particle number $n+1$ ) at grid position $m$. This process implies a change in the free energy of the system $\Delta F_{m}$ given by Eq. (9) and a change in the chemical potential of the gas as a result of increasing the number of molecules from $n$ to $n+1$. Therefore, the process of insertion will have a statistical weight given by

$\sigma_{m, i ; n \rightarrow n+1}=\exp \left[-\frac{\mu(n+1)-\mu(N)-\Delta F_{m}}{k T}\right]$

where $\mu$ is the chemical potential of the gas and $N$ is the number of molecules at equilibrium under a pressure $p$. Taking into account that $\mu=\mu+k T \ln c$, where $c$ is the gas concentration, Eq. (14) can be written as

$\sigma_{m, i ; n \rightarrow n+1}=\frac{N}{n+1} \exp \left(\frac{-\Delta F_{m}}{k T}\right)$

By the same token, the statistical weight associated with the removal of a particle of gas from the cell $m$ is given by

$\sigma_{m, i ; n \rightarrow n-1}=\frac{n-1}{N} \exp \left(\frac{\Delta F_{m}}{k T}\right)$

The number of molecules at equilibrium, $N$, depends on pressure and volume so that Eqs. (15) and (16) can be written as

$\sigma_{m, i ; n \rightarrow n+1}=\frac{p V}{(n+1) k T} \exp \left(\frac{-\Delta F_{m}}{k T}\right)$

and

$\sigma_{m, i ; n \rightarrow n-1}=\frac{k T(n-1)}{p V} \exp \left(\frac{\Delta F_{m}}{k T}\right)$
An additional statistical weight is associated with the volume of the molecule of the gas. Since the number of grid positions occupied by the volume $V_{\text {mol }}$ of each molecule inserted in the cubic box is $g=V_{\text {mol }}(G / L)^{3}$, where $L$ and $G$ are, respectively, the side length of the cubic box and the number of intervals in which it is divided, the whole volume of the matrix will be completely filled when we succeed in 1 out of every grid position during sampling. The geometric statistical weight associated with this effect can be defined as

$\sigma_{i ; n \rightarrow n+1}^{\prime}=\frac{1}{g}=\frac{L^{3}}{G^{3} V_{\mathrm{mol}}}$

For removal:

$\sigma_{r ; n \rightarrow n-1}^{\prime}=g=\frac{G^{3} V_{\mathrm{mol}}}{L^{3}}$

It is worthy noting that at low pressures the equilibrium number of particles of an ideal gas in the cubic box, under STP conditions, may be smaller than 1 . To avoid this inconvenience we set up an ensemble of $\left(N_{\text {box }}\right)^{3}$ boxes, in this simulation $6^{3}$ boxes, identical to that containing the polymer matrix, packed within a cube with side $L N_{\text {box }}$. Then the volume appearing on Eqs. (19) and (20) is $\left(L N_{\text {box }}\right)^{3}$, while each grid position is repeated $\left(N_{\text {box }}\right)^{3}$ times with periodic boundary conditions within the ensemble. The overall statistical weight associated with the insertion of a molecule at the $m$ grid position of the box of volume $\left(L N_{\text {box }}\right)^{3}$ already containing $n$ molecules of gas is

$\sigma_{i ; n \rightarrow n+1}=\sigma_{m, i ; n \rightarrow n+1} \sigma_{i ; n \rightarrow n+1}^{\prime}$

On the other hand, the overall statistical weight of removal of the molecule of gas is

$\sigma_{i ; n \rightarrow n-1}=\sigma_{m, i ; n \rightarrow n-1} \sigma_{i ; n \rightarrow n-1}^{\prime}$

The probability of insertion and removal of a gas molecule in the cubic box can be obtained from the normalization of the statistical weight of Eqs. (21) and (22), that is

$p_{i ; n \rightarrow n+1}=\frac{\sigma_{i ; n \rightarrow n+1}}{\sigma_{i ; n \rightarrow n+1}+\sigma_{i ; n \rightarrow n-1}}$

$p_{i ; n \rightarrow n-1}=\frac{\sigma_{i ; n \rightarrow n-1}}{\sigma_{i ; n \rightarrow n+1}+\sigma_{i ; n \rightarrow n-1}}$

Insertion and removal of gas molecules in the polymer were carried out using Monte Carlo techniques. The Monte Carlo simulation of the sorption process consisted in 50 series of $5 \times 10^{6}$ cycles. At each cycle, a grid position $m$ within the polymer matrix having a volume $\left(L N_{\text {box }}\right)^{3}$ was randomly selected. The value of the energy at that position, $F_{m}$, together with the number of particles, $n$, previously loaded into the system allows the evaluation of the probabilities of insertion $p_{i, m}$ and removal $p_{r, m}$ at grid point $m$ according to Eqs. (23) and (24). Then, a random number $x$ within the interval $0-1$ was generated and compared with $p_{i, m}$. When $x \leq p_{i, m}$, an attempt to insert a new particle into position $m$ was performed, otherwise, i.e. when $x>p_{i, m}$, removal of one particle from position $m$ was attempted.

An insertion attempt was successful, i.e. a new particle was inserted with its center of masses in position $m$, when none of 
Table 4

Values of the free volume in the cubic box determined by Eq. (25) (method 1) and Eq. (26) (method 2)

\begin{tabular}{lll}
\hline Matrix & $f_{\mathrm{V}}$, method 1 $(\%)$ & $f_{\mathrm{V}}$, method 2 $(\%)$ \\
\hline Poly-PhNDI & 19.72 & 20.08 \\
Poly-HPhNDI & 18.58 & 18.40
\end{tabular}

the $n$ particles previously loaded into the system has its center of masses within a distance smaller than a molecular diameter from position $m$, i.e. when the new loaded molecule would not overlap with any of the previously loaded particles. However, insertion attempts that would place the new molecule overlapping with a previously loaded one are very infrequent because the number of molecules loaded into the matrix is much smaller than what would be allowed by the ratio among the volumes of the matrix and the molecule. Consequently, most of the insertion attempts take place at well-separated points. For this reason, no interactions among molecules of solute were considered when computing the exponential of energy appearing in Eqs. (17) and (18) except the hard spheres potential, which is implicit in the fact of avoiding overlapping among molecules of solute. On the other hand, a removal attempt was successful, i.e. one particle was removed from the system when its center of masses lies within a distance smaller than the molecular radius from the tested position, i.e. when the tested position is one among the $\mathrm{g}$ grid positions occupied by one of the molecules contained in the system. Of course, failed attempts to either insert or remove particles leave the system unchanged.

Two batches of sorption simulations were made. In the first batch, the volume $V$ used in Eqs. (17) and (18) was that of the cubic box while in the second one, the volume used was the free volume, that is $f_{\mathrm{V}}=V-\sum_{i}^{n} V_{\mathrm{vdW} ; i}$ where $V_{\mathrm{vdW} ; i}$ is the van der Waals volume of atom $i$ contained in the cubic box of volume $V$. The values of the free volume fraction (Table 4$), f_{\mathrm{V}}$ was calculated by the two methods indicated by the following expressions:

$f_{\mathrm{V}}=\frac{\text { unoccupied sites }}{\left(N_{\mathrm{box}}\right)^{3}}$

$f_{\mathrm{V}}=V_{\text {cell }}-V_{\text {atoms }}$

where $V_{\text {cell }}$ is the cell volume and $V_{\text {atoms }}$ is the van der Waals volume occupied by the atoms of the matrix.

Values of the experimental solubility coefficients for different gases in PNDCI and H-PNDCI membranes are shown in the second column of Tables 5 and 6 , respectively. The second and third columns of the tables present the simulated values for the first and second batch simulations. With the exception of carbon dioxide, the solubility coefficients simulated for the gases are in fair agreement with the experimental results independently on whether $V$ or $f_{\mathrm{V}}$ are used in Eqs. (17) and (18). The strong discrepancy between experimental and simulated values of the solubility coefficient of $\mathrm{CO}_{2}$ in the polymers could be attributed to plasticizing effects that enhance the solubility of these species.
Table 5

Experimental and simulated values of the solubility coefficient for different gase in PNDCI membranes, at $30^{\circ} \mathrm{C}$ and 1 bar

\begin{tabular}{lccl}
\hline Gas & $10^{3} \times S_{\text {exp }}$ & $10^{3} \times S_{\text {simul }}^{*}$ & $10^{3} \times S_{\text {simul }}^{* *}$ \\
\hline $\mathrm{H}_{2}$ & 0.8 & 1.4 & 0.5 \\
$\mathrm{~N}_{2}$ & 1.4 & 2.9 & 1.1 \\
$\mathrm{O}_{2}$ & 2.3 & 4.4 & 1.8 \\
$\mathrm{CO}_{2}$ & 63.2 & 11.4 & 5.7 \\
$\mathrm{CO}$ & 3.6 & 3.5 & 1.5 \\
$\mathrm{CH}_{4}$ & 7.5 & 8.8 & 4.6
\end{tabular}

Single and double stars indicated solubility coefficients simulated using for $V$ in Eqs. (17) and (18) the volume of the cubic box and the free volume, respectively. The values of the solubility coefficient are given in $\mathrm{cm}^{3}(\mathrm{STP}) / \mathrm{cm}^{3} \mathrm{~cm} \mathrm{Hg}$.

Table 6

Experimental and simulated values of the solubility coefficient for different gases in $\mathrm{H}$-PNDCI, at $30^{\circ} \mathrm{C}$ and 1 bar

\begin{tabular}{lccl}
\hline Gas & $10^{3} \times S_{\text {exp }}$ & $10^{3} \times S_{\text {simul }}^{*}$ & $10^{3} \times S_{\text {simul }}^{* *}$ \\
\hline $\mathrm{H}_{2}$ & 0.6 & 1.5 & 0.5 \\
$\mathrm{~N}_{2}$ & 1.3 & 3.8 & 1.5 \\
$\mathrm{O}_{2}$ & 2.1 & 5.3 & 2.1 \\
$\mathrm{CO}_{2}$ & 62.6 & 13.7 & 7.2 \\
$\mathrm{CO}$ & 2.8 & 4.5 & 1.8 \\
$\mathrm{CH}_{4}$ & 8.3 & 10.8 & 6.01
\end{tabular}

Single and double stars indicated solubility coefficients simulated using for $V$ in Eqs. (17) and (18) the volume of the cubic box and the free volume, respectively. The values of the solubility coefficient are given in $\mathrm{cm}^{3}(\mathrm{STP}) / \mathrm{cm}^{3} \mathrm{~cm} \mathrm{Hg}$.

Simulated curves showing the pressure dependence of the solubility coefficient for PNDCI and $\mathrm{H}-\mathrm{PNDCI}$, at $30^{\circ} \mathrm{C}$, are shown in Figs. 7 and 8, respectively. In the inset the curves simulated using the free volume in Eqs. (17) and (18) are shown. It can be seen that the isotherms undergo a sharp decrease at low pressure reaching a nearly constant value at moderate pressures. This behavior experimentally found for the pressure dependence of the gas solubility of glassy polymers has traditionally been interpreted in terms of the dual mode model that assumes the polymer matrix as made up of a continuous phase where gas absorp-

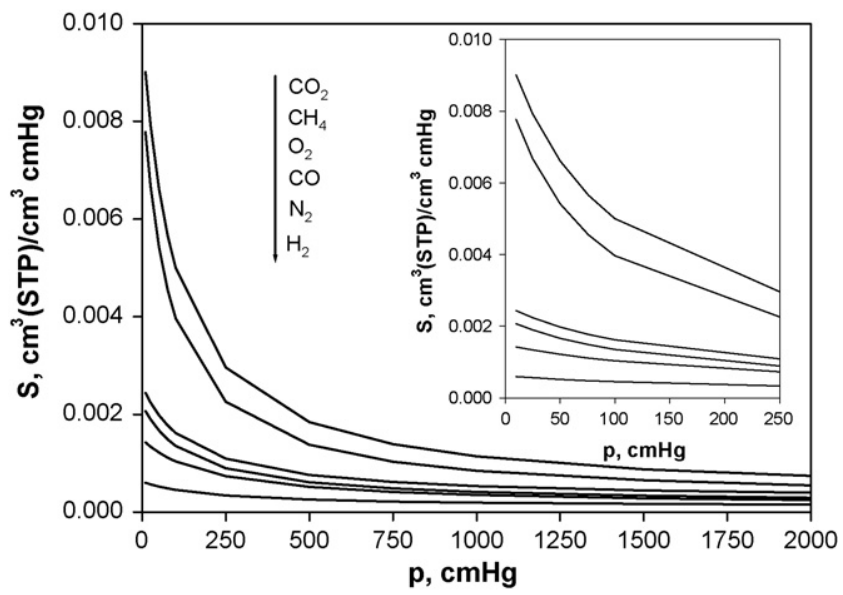

Fig. 7. Variation of the simulated solubility coefficient with pressure of different gases in PNDCI membranes at $30^{\circ} \mathrm{C}$. Inset: the simulations were performed using the free volume instead of the volume of the cubic box. 


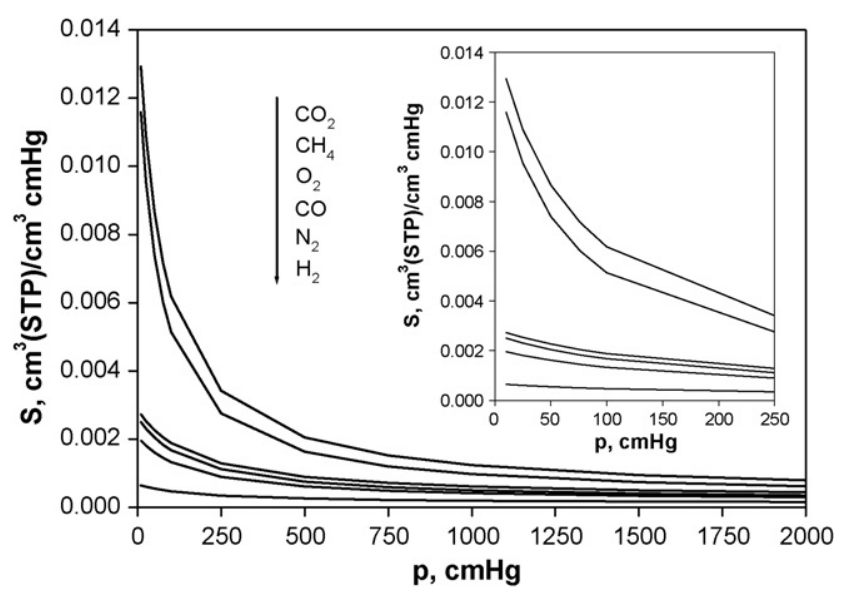

Fig. 8. Variation of the simulated solubility coefficient of gases in poly-HNDI membranes at $30^{\circ} \mathrm{C}$. The curves in the inset were computed using the free volume instead of the volume of the cubic cell.

tion occurs. Microcavities that account for the volume excess in glassy polymers disperse in the continuous phase act like Langmuir sites where adsorption processes take place. According to the model, the pressure dependence of the solubility coefficient is given by

$S=k_{\mathrm{D}}+\frac{C_{\mathrm{H}}^{\prime} b}{1+b p}$

where $k_{\mathrm{D}}$ is Henry's constant, $C_{\mathrm{H}}^{\prime}$ is the concentration of gas in Langmuir sites whereas $b$ is a parameter related with the affinity gas polymer. The isotherms computed using $V$ and $f_{\mathrm{V}}$ are nearly similar though the former are slightly shifted vertically to higher values owing to the fact that the values of $k_{\mathrm{D}}$ obtained with $V$ are higher than those obtained with $f_{\mathrm{V}}$. Eq. (27) fits to the computed isotherms giving reasonable values for the Henry's solubility constant though the gas concentration in Langmuir sites seems to be underestimated. As an example, the values of $k_{\mathrm{D}}, C_{\mathrm{H}}^{\prime}$ and $b$ for $\mathrm{O}_{2}$ in PNDCI obtained using the volume of the cubic box in the computation are, $4.0 \times 10^{-4} \mathrm{~cm}^{3}(\mathrm{STP}) / \mathrm{cm}^{3} \mathrm{~cm} \mathrm{Hg}$, $0.9 \mathrm{~cm}^{3}(\mathrm{STP}) / \mathrm{cm}^{3}$ and $7.2 \times 10^{-3}\left(\mathrm{~cm} \mathrm{Hg}^{-1}\right.$, respectively. If the free volume is used, then the respective values in the units indicated are $2.4 \times 10^{-4}, 0.3$ and $7.0 \times 10^{-3}$. For H-PNDCI the dual mode parameters for oxygen are $3.9 \times 10^{-4}, 7.0$ and 0.3 using $V$ in the computations and $2.3 \times 10^{-4}, 1.0$ and 6.0 if $f_{\mathrm{V}}$ is used. Notice that in all the cases the values simulated for $C_{\mathrm{H}}^{\prime}$ are rather small if the experimental values reported for other systems with high glass transition temperature are taken as a basis of comparison [48].

In Table 7 the values of the permeability coefficients, at $30^{\circ} \mathrm{C}$ and $1 \mathrm{bar}$, simulated using $V$ and $f_{\mathrm{V}}$ for the computation of the solubility coefficient are compared with the experimental ones. An inspection of the data shows a rather good agreement between experimental and simulated values for all the gases, with the exception of carbon dioxide. In this latter case, the permeability coefficient is more than one order of magnitude below the experimental one as a consequence of the low solubility coefficient obtained by simulation. The same behavior was obtained by Fried et al. [70] and Heuchel et al. [71] in
Table 7

Comparison between the experimental permeability coefficient, $P_{\exp }$, and the simulated results using the volume $V, P^{*}$, and the free volume, $P^{* *}$, for the simulations of the solubility coefficient

Gas PNDCI membranes

\begin{tabular}{cccllll}
\hline $\begin{array}{l}P_{\text {exp }} \\
\text { (barrers) }\end{array}$ & $\begin{array}{l}P^{*} \\
\text { (barrers) }\end{array}$ & $\begin{array}{l}P^{* *} \\
\text { (barrers) }\end{array}$ & $\begin{array}{l}P_{\text {exp }} \\
\text { (barrers) }\end{array}$ & $\begin{array}{l}P^{*} \\
\text { (barrers) }\end{array}$ & $\begin{array}{l}P^{* *} \\
\text { (barrers) }\end{array}$ \\
\hline 11.0 & 40.23 & 14.30 & 7.22 & 24.6 & 8.40 \\
0.31 & 0.31 & 0.12 & 0.12 & 0.38 & 0.15 \\
1.44 & 0.71 & 0.28 & 0.66 & 0.81 & 0.31 \\
11.44 & 0.79 & 0.40 & 4.51 & 0.30 & 0.16 \\
0.52 & 0.37 & 0.15 & 0.21 & 0.24 & 0.10 \\
0.54 & 0.51 & 0.26 & 0.15 & 0.35 & 0.19
\end{tabular}

The results were measured and computed at $T=300 \mathrm{~K}$ and $p=1 \mathrm{bar}$.

poly(2,6-dimethyl-1,4-phenylene oxide) and polyimide membranes, respectively. As indicated above, plasticizing effects not accountable for in simulations seems to be the cause of the considerable discrepancy between the simulated and experimental values of the permeability coefficient of carbon dioxide in PNDCI and H-PNDCI membranes. However, the rather good agreement between the simulated and experimental results for the permeability coefficients of the other gases encourages the use of the TS approach for diffusion and the modified Widom method for sorption as valuable tools to predict gas permeation as a function of the chemical structure of glassy membranes.

\section{Conclusions}

An inspection of the experimental and simulated diffusion coefficients of the gases shows that hydrogenation of the double bonds of the norbornene residues hinders gas transport in the $\mathrm{H}$ PNDCI membranes presumably as consequence of increasing packing volume efficiency facilitated by chain mobility of the saturated double bonds.

The pressure dependence of the simulated solubility coefficient follows the same trends observed in the sorption processes in glassy membranes. In general the values of the solubility coefficients computed using the total volume of the box cell are two to three times higher than those computed utilizing the free volume.

Agreement between simulated and experimental results for all the magnitudes studied in the present work is pretty good, with the noteworthy exception of $\mathrm{CO}_{2}$, and specially its solubility coefficients which is substantially underestimated by the simulation. However, at this moment, we cannot provide any reasonable explanation for such disagreement in the case of this particular molecule.

\section{Acknowledgments}

This work was supported by Comunidad de Madrid (CAM projects: GR/MAT/0810/2004; S-0505/MAT/0227) and CICYT (projects: MAT-2005-05648-C02-01, CTQ2005-04710/BQU; MAT2004-01347). 


\section{Nomenclature}

A permeation area $\left(\mathrm{cm}^{2}\right)$

$b \quad$ parameter related with the gas-polymer affinity $\left((\mathrm{cm} \mathrm{Hg})^{-1}\right)$

$c \quad$ concentration $\left(\mathrm{cm}^{3}(\mathrm{STP}) / \mathrm{cm}^{3}\right)$

$C$ normalization constant

$C_{\mathrm{H}}^{\prime} \quad$ gas concentration in Langmuir sites

$\left(\mathrm{cm}^{3}(\mathrm{STP}) / \mathrm{cm}^{3}\right)$

$D$ diffusion coefficient $\left(\mathrm{cm}^{2} / \mathrm{s}\right)$

E energy

$f_{\mathrm{V}} \quad$ free volume in the $\mathrm{PBC}$ box

$F \quad$ Hemholtz free energy

$g \quad$ number of grid cells occupied by the volume of a gas molecule in the PBC box

$G \quad$ number of intervals in which $L$ is divided

$k \quad$ Boltzmann's constant

$k_{\mathrm{D}} \quad$ Henry's solubility coefficient

$\left(\mathrm{cm}^{3}(\mathrm{STP}) / \mathrm{cm}^{3} \mathrm{~cm} \mathrm{Hg}\right)$

$l \quad$ thickness of the membrane $(\mathrm{cm})$

$L \quad$ side length of the PBC box $(\AA)$

$m \quad$ mass of the gas particle

$n \quad$ number of particles of the gas in the PBC box out of equilibrium under a pressure $p$

$N \quad$ number of particles of the gas in the PBC box at equilibrium under a pressure $p$

$p \quad$ pressure of the gas in the downstream chamber $(\mathrm{cm} \mathrm{Hg})$

$p_{i} \quad$ probability of insertion or removal of a gas particle in the cell $i$

$p_{0} \quad$ pressure of the gas in the upstream chamber (cm Hg)

$P \quad$ permeability coefficient $($ barrer $=$ $\left.10^{-10} \mathrm{~cm}^{3}(\mathrm{STP}) \mathrm{cm} /\left(\mathrm{cm}^{2} \mathrm{~s} \mathrm{~cm} \mathrm{Hg}\right)\right)$

PBC periodic boundary conditions

$r \quad$ vector position $(\mathrm{cm})$

$R_{i j} \quad$ rate of passage of a gas particle from site $i$ to $j$ in the PBC box

$\mathbf{R}_{m} \quad$ vector joining the center of mass of cell $m$ with the equilibrium position of the atom $i$ of the matrix in the PBC box

$S \quad$ solubility coefficient $\left(\mathrm{cm}^{3}(\mathrm{STP}) / \mathrm{cm}^{3} \mathrm{~cm} \mathrm{Hg}\right)$

$t \quad$ time (s)

$T \quad$ temperature (K)

$V \quad$ volume $\left(\mathrm{cm}^{3}\right)$

$W(\cdots)$ probability of finding an atom of the matrix in the PBC box in the conditions indicated between brackets

Z partition function

\section{Greek letters}

$\gamma \quad$ weighting factor

$\delta \quad$ deviation of an atom of the polymeric matrix in the PBC box from the equilibrium position

$\Delta$ $\theta \quad$ time lag (s) (in experimental part); orientational angle $\left(^{\circ}\right)$ (in simulations)

$\mu \quad$ chemical potential

$\sigma \quad$ statistical weight; van der Waals parameter $(\AA)$ in Table 3

$\tau$

residence time (s)

\section{References}

[1] R.F. Kesting, A.K. Fritzsch, Polymeric Gas Separation Membranes, WileyInterscience, New York, 1993.

[2] Y. Yampolskii, I. Pinnau, B.D. Freeman, Materials Science of Membranes for Gas and Vapor Separation, Wiley-Interscience, New York, 2006.

[3] M. Aguilar-Vega, D.R. Paul, Gas-transport properties of poly(2,2,4,4tetramethyl cyclobutane carbonate), Polym. Sci.: Phys. Ed. 31 (1993) 991.

[4] K. Ghosal, R.T. Chern, Aryl-nitration of poly(phenylene oxide) and polysulfone: structural characterization and gas permeability, J. Membr. Sci. 72 (1992) 91.

[5] M.B. Moe, W.J. Koros, D.R. Paul, Effects of molecular-structure and thermal annealing on gas-transport in 2-tetramethyl bisphenol-a polymer, J. Polym. Sci.: Phys. Ed. 26 (1988) 1931.

[6] M.W. Hellums, W.J. Koros, G.R. Husk, D.R. Paul, Study of microporous membrane structure by image analysis, J. Membr. Sci. 46 (1989) 93

[7] N. Maruganandam, D.R. Paul, Evaluation of substituted polycarbonates and a blend with polystyrene as gas separation membranes, J. Membr. Sci. 34 (1987) 185.

[8] J.S. McHattie, W.J. Koros, D.R. Paul, Effect of isopropylidene replacement on gas-transport properties of polycarbonates, J. Polym. Sci.: Phys. Ed. 29 (1991) 731.

[9] M.W. Hellums, W.J. Koros, J.C. Schmidhauser, Gas separation properties of spirobiindane polycarbonate, J. Membr. Sci. 67 (1992) 75.

[10] J.S. McHattie, W.J. Koros, D.R. Paul, Gas transport properties of polysulphones. 2. Effect of bisphenol connector groups, Polymer 32 (1991) 2618.

[11] J.S. McHattie, W.J. Koros, D.R. Paul, Gas transport properties of polysulphones. 1. Role of symmetry of methyl group placement on bisphenol rings, Polymer 32 (1991) 840.

[12] C.L. Aitken, W.J. Koros, D.R. Paul, Effect of structural symmetry on gas transport properties of polysulfones, Macromolecules 25 (1992) 3424

[13] C.L. Aitken, W.J. Koros, D.R. Paul, Gas transport properties of biphenol polysulfones, Macromolecules 25 (1992) 3651.

[14] C.L. Aitken, D.R. Paul, D.K. Mohanty, Gas-transport properties of poly(arylether bissulfone)s and poly(arylether bisketone)s, J. Polym. Sci.: Phys. Ed. 31 (1993) 983.

[15] C.L. Aitken, D.R. Paul, Gas transport properties of polysulfones based on dihydroxynaphthalene isomers, J. Polym. Sci.: Phys. Ed. 31 (1993) 1061.

[16] K. Ghosal, R.T. Chern, B.D. Freeman, Gas-permeability of radel-a polysulfone, J. Polym. Sci.: Phys. Ed. 31 (1993) 891.

[17] J.S. McHattie, W.J. Koros, D.R. Paul, Gas transport properties of polysulphones. 3. Comparison of tetramethyl-substituted bisphenols, Polymer 33 (1992) 1701.

[18] M.R. Coleman, W.J. Koros, Isomeric polyimides based on fluorinated dianhydrides and diamines for gas separation applications, J. Membr. Sci. 50 (1990) 285.

[19] T.H. Kim, W.J. Koros, G.R. Husk, Advanced gas separation membrane materials: rigid aromatic polyimides, Sep. Sci. Technol. 23 (1988) 1611.

[20] T.H. Kim, W.J. Koros, G.R. Husk, K.C. O’Brien, Relationship between gas separation properties and chemical structure in a series of aromatic polyimides, J. Membr. Sci. 37 (1988) 45

[21] S.A. Stern, Y. Mi, H. Yamamoto, A.K. St. Clair, Structure permeability relationships of polyimide membranes-applications to the separation of gas-mixtures, J. Polym. Sci.: Phys. Ed. 27 (1989) 1887.

[22] K.C. O'Brien, W.J. Koros, G.R. Husk, Sorption of benzene and $N$-hexane in polyethylene, J. Membr. Sci. 35 (1988) 217. 
[23] K. Tanaka, M. Okano, H. Toshino, H. Kita, K. Okamoto, Effect of methyl substituents on permeability and permselectivity of gases in polyimides prepared from methyl-substituted phenylenediamines, J. Polym. Sci. Part B: Polym. Phys. Ed. 30 (1992) 907.

[24] H. Kita, T. Inada, K. Tanaka, K. Okamoto, Effect of photocrosslinking on permeability and permselectivity of gases through benzophenonecontaining polyimide, J. Membr. Sci. 87 (1994) 139.

[25] L. Yang, J. Fang, N. Meichin, K. Tanaka, H. Kita, K. Okamoto, Gas permeation properties of thianthrene-5,5,10,10-tetraoxide-containing polyimides, Polymer 42 (2001) 2021.

[26] M. Wessling, T.V.D. Boomgard, M.H.V. Mulder, C.A. Smolders, Makromol. Chem. Makromol. Symp. 70/71 (1993) 379.

[27] E. Herisema, Polyoxadiazoles and Polytriazoles Gas Separation Membranes, Ph.D. Thesis, Enschede, The Netherlands, 1991.

[28] F.R. Sheu, R.T. Chern, V.T. Stannet, H.B. Hopfenberg, Transport of carbondioxide and methane in glassy aromatic polyesters, J. Polym. Sci.: Phys. Ed. 26 (1988) 883

[29] R.T. Chern, N.F. Brown, Solubilities and diffusivities of permanent gases in poly(phenolphthalein terephthalate), Macromolecules 23 (1990) 2370.

[30] F.R. Sheu, R.T. Chern, Effects of packing density on the gas-transport properties of poly(phenolphthalein phthalate)s, J. Polym. Sci.: Phys. Ed. 27 (1989) 1121.

[31] R.T. Chern, Repeat unit structure and gas transport properties of aromatic polymers, Sep. Sci. Technol. 25 (1990) 1325.

[32] S.J. Charati, A.Y. Houde, S.S. Kulkarni, M.J. Kulkarni, Transport of gases in aromatic polyesters—correlation with waxd studies, J. Polym. Sci.: Phys. Ed. 29 (1991) 921

[33] M. Aguilar-Vega, D.R. Paul, Gas-transport properties of polyphenylene ethers, J. Polym. Sci.: Phys. Ed. 31 (1993) 1577.

[34] J. Zhang, X. Hou, Characterization of hydrogen-permselective microporous ceramic membranes, J. Membr. Sci. 97 (1994) 275.

[35] D.N. Theodorou, Principles of Molecular Simulation of Gas Transport in Polymers, in Ref. 2, Chapter 2 (and references therein).

[36] J.R. Fried, Molecular Simulation of Gas and Vapor Transport in Highly Permeable Membranes, in Ref. 2, Chapter 3 (and references therein).

[37] R.M. Sok, H.J.C. Berendsen, W.F. van Gunsteren, Molecular dynamics simulation of the transport of small molecules across a polymer membrane, J. Chem. Phys. 96 (1992) 4699.

[38] F. Müller-Plathe, Diffusion of penetrants in amorphous polymers: a molecular dynamics study, J. Chem. Phys. 94 (1991) 3192.

[39] A.A. Gusev, F. Müller-Plathe, W.F. Laaksonen, W.F. van Gunsteren, U.W. Suter, Dynamics of small molecules in bulk polymers, Adv. Polym. Sci. 116 (1994) 207.

[40] B. Widom, Some topics in theory of fluids, J. Chem. Phys. 39 (1963) 2808.

[41] E. Saiz, M.M. López-González, E. Riande, J. Guzmán, V. Compañ, Simulations of diffusive and sorption processes of gases in polyimide membranes: comparison with experiments, Phys. Chem. Chem. Phys. 5 (2003) 2862.

[42] P. Tiemblo, E. Saiz, J. Guzmán, E. Riande, Comparison of simulated and experimental transport of gases in commercial poly(vinyl chloride), Macromolecules 35 (2002) 4167.

[43] E.Sh. Finkel'shtein, M.L. Gringolts, N.V. Ushakov, V.G. Lakhtin, S.A. Soloviev, Y.P. Yampol'skii, Synthesis and gas permeation properties of new ROMP polymers from silyl substituted norbornadienes and norbornenes, Polymer 44 (2003) 2843.

[44] B.R. Wilks, W.J. Chung, P.J. Ludovice, M.R. Rezc, P. Meakin, Impact of average free-volume element size on transport in stereoisomers of polynorbornene. I. Properties at $35^{\circ} \mathrm{C}$, J. Polym. Sci. Part B: Polym. Phys. 41 (2003) 2185 .

[45] B.R. Wilks, M.E. Rezac, Impact of average free-volume element size on transport in stereoisomers of polynorbornene. II. Impact of temperature on solubility, J. Polym. Sci. Part B: Polym. Phys. 41 (2003) 1939.

[46] V.I. Bondar, Y.M. Kukharskii, Yu.P. Yampol'skii, E.Sh. Finkel'shtein, K.L. Makovetskii, Permeation and sorption in polynorbornenes with organosilicon substituents, J. Polym. Sci.: Phys. Ed. 31 (1993) 1273.

[47] H. Chien-Chieh, C. Chin-Shun, R. Ruoh-Chyu, L. Juin-Yih, Effect of free volume and sorption on membrane gas transport, J. Membr. Sci. 226 (2003) 51.
[48] Y.P. Yampol'skii, N.B. Bespalova, E.S. Finkel'shtein, V.I. Bondar, A.V. Popov, Synthesis, gas permeability, and gas sorption properties of fluorinecontaining norbornene polymers, Macromolecules 27 (1994) 2872.

[49] Y.K. Kawakami, H. Toda, M. Higashino, Y. Yamashita, Polynorbornenes with oligodimethylsiloxanyl substituents for selectively oxygen permeable membrane material, Polym. J. 20 (1988) 285.

[50] V.V. Teplyakov, D.R. Paul, N.B. Bespalova, E.Sh. Finkelshtein, Gas permeation in a fluorine-containing polynorbornene, Macromolecules 25 (1992) 4218.

[51] T. Steinhäuser, W. Koros, Gas permeation and sorption studies on stereoregular polynorbornene, J. Polym. Sci. Part B: Polym. Phys. 35 (1997) 91.

[52] K.D. Dorkenoo, P.H. Pfromm, M.E. Rezac, Gas transport properties of a series of high Tg-polynorbornenes with aliphatic pendant groups, J. Polym. Sci. Part B: Polym. Phys. 36 (1998) 797.

[53] Ch. Zhao, M.R. Ribeiro, M.N. Pinho, V.S. Subrahmanyam, C.L. Gil, A.P. de Lima, Structural characteristics and gas permeation properties of polynorbornenes with retained bicyclic structure, Polymer 42 (2001) 2455.

[54] A. Pineda-Contreras, M.A. Tlenkopatchev, M.M. López-González, E. Riande, Synthesis and gas transport properties of new high glass transition temperature ring-opened polynorbornenes, Macromolecules 35 (2002) 4677.

[55] M.A. Tlenkopatchev, J. Vargas, M.M. López-González, E. Riande, Gas transport in polymers prepared via metathesis copolymerization of exo- $\mathrm{N}$-phenyl-7-oxanorbornene-5,6-dicarboximide and norbornene, Macromolecules 36 (2003) 8483.

[56] M. Tlenkopatchev, J. Vargas, M.A. Girón, M. López-González, E. Riande, Gas sorption in new fluorine containing polynorbornenes with imide side chain groups, Macromolecules 38 (2005) 2696.

[57] J. Vargas, A.A. Santiago, M.A. Tlenkopatchev, R. Gaviño, M.Fe. Laguna, M. López-González, E. Riande, Gas transport and ionic transport in membranes based on polynorbornenes with functionalized imide side groups, Macromolecules 40 (2007) 563.

[58] R.M. Barrer, Trans. Faraday Soc. 35 (1939) 628.

[59] A.A. Gusev, U.W. Suter, D.J. Moll, Relationship between helium transport and molecular motions in a glassy polycarbonate, Macromolecules 28 (1995) 2582

[60] A.A. Gusev, S. Arizzi, U.W. Suter, D.J. Moll, Dynamics of light gases in rigid matrices of dense polymers, J. Chem. Phys. 99 (1993) 2221.

[61] A.A. Gusev, U.W. Suter, Dynamics of small molecules in dense polymers subject to thermal motion, J. Chem. Phys. 99 (1993) 2228.

[62] A.A. Gusev, U.W. Suter, Comput-aided mate, Design 1 (1993) 63.

[63] M. López-González, E. Saiz, J. Guzmán, E. Riande, Experimental and simulation studies on the transport of gaseous diatomic molecules in polycarbonate membranes, J. Chem. Phys. 115 (2001) 6728.

[64] M.F. Laguna, J. Guzmán, E. Riande, E. Saiz, Experimental and simulation studies on the transport of argon through poly(pentaerythritoltribenzoate acrylate), Macromolecules 31 (1998) 7488.

[65] Materials Studio 3.2, VISUALIZER, AMORPHOUS CELL and DISCOVER modules, Aceelrys Inc., San Diego, CA, USA, 2005.

[66] H. Sun, S.J. Mumby, J.R. Maple, A.T. Hagler, An ab-initio cff 93 all-atom force-field for polycarbonates, J. Am. Chem. Soc. 116 (1994) 2978.

[67] P.P. Ewald, Berechnung optischer und elektrostatishe gitterpotentiale, Ann. Physik 64 (1921) 253.

[68] M.F. Laguna, J. Guzmán, E. Saiz, E. Riande, Experimental and theoretical studies on the permeation of argon through matrices of acrylic polymers containing 1,3-dioxane groups in their structure, J. Chem. Phys. 110 (1999) 3200 .

[69] E. Kucukpinar, P. Doruker, Molecular simulations of small gas diffusion and solubility in copolymers of styrene, Polymer 44 (2003) 3607.

[70] J.R. Fried, M. Sadat-Akhavi, J.E. Mark, Molecular simulation of gas permeability: poly(2,6-dimethyl-1,4-phenylene oxide), J. Membr. Sci. 149 (1998) 115.

[71] M. Heuchel, D. Hofmann, P. Pullumbi, Molecular modeling of smallmolecule permeation in polyimides and its correlation to free-volume distributions, Macromolecules 37 (2004) 201. 